\title{
Motif statistics and spike correlations in neuronal networks
}

Yu Hu ${ }^{1 *}$, James Trousdale ${ }^{2}$, Krěsimir Josić 2,3 , Eric Shea-Brown ${ }^{1,4}$

From Twenty First Annual Computational Neuroscience Meeting: CNS*2012

Decatur, GA, USA. 21-26 July 2012

Motifs are patterns of subgraphs that are the building blocks of complex networks. Recent experiments have characterized the frequencies with which different motifs occur in biological neural networks, and found remarkable deviations from what we would expect if the networks were randomly connected [1]. Here, we study
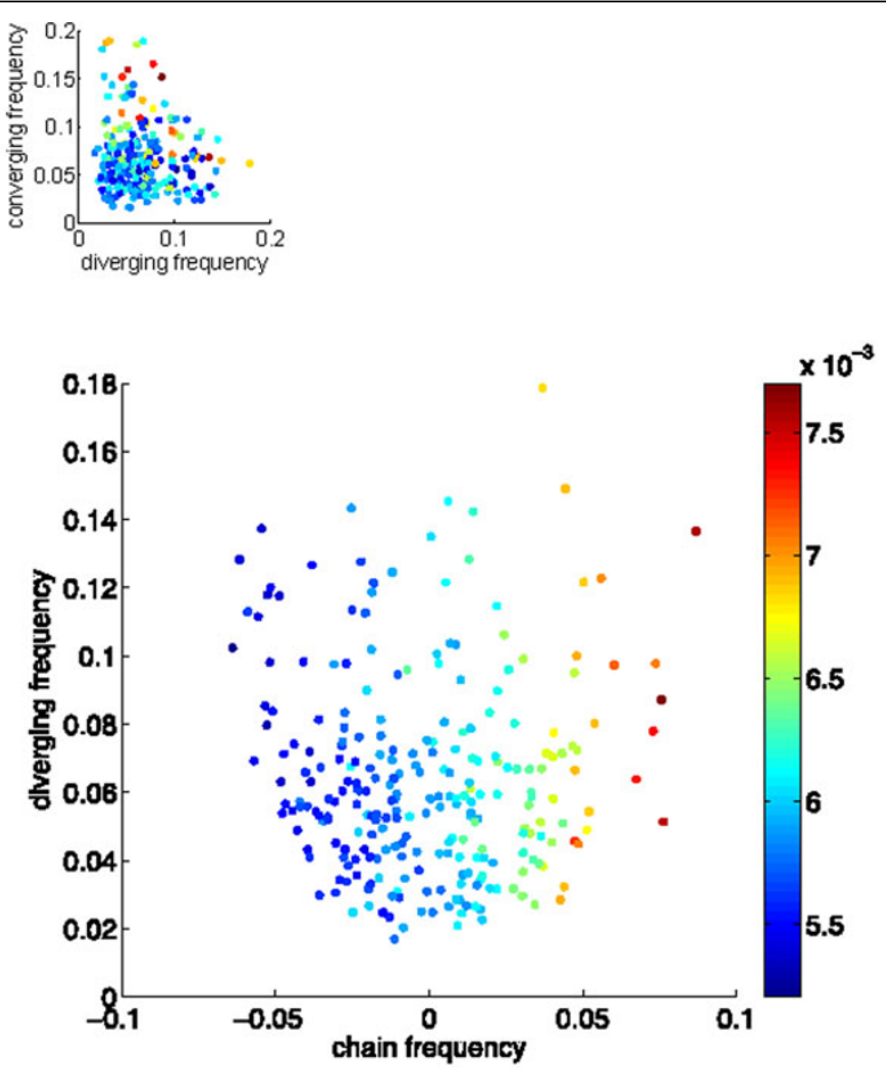

Figure 1 Each dot represent one network sample plotted against its chain and diverging motif frequencies. Color shows the standard deviation of correlations in the network. Inset is the same plot with respect to diverging and converging motifs.

\footnotetext{
* Correspondence: huyu@uw.edu

'Department of Applied Mathematics, University of Washington, Seattle, WA 98195, USA

Full list of author information is available at the end of the article
} 
the impact of such patterns of connectivity on the level of correlated, or synchronized, spiking activity among pairs of cells. Correlations in spiking activity have been shown to strongly impact the neural coding of information.

We use a linear, stochastic model of recurrent networks. A cell's time-dependent firing rate is perturbed from its baseline level by convolution of a response kernel and the input signal from presynaptic neurons. Each neuron generates spikes as an inhomogeneous Poisson process. Previous studies have shown that such models can capture pairwise correlations in integrate and fire networks $[2,3]$, and they are closely related to spike response and Hawkes models $[4,6]$.

For this model, there is an explicit expression for pairwise correlation in terms of the connectivity matrix. By expanding this expression in a series, one can relate each term to a different motif (with a different number of connections). Through a resumming technique, we show that the average correlation across the network can be closely approximated using the frequencies of only first and second order motifs. These are the diverging motif-two cells both receiving projections from another cell, its counterpart the converging motif-two cells projecting to a common cell, and the chain motifthree cells linked by two consecutive projections. Specifically, we show that the prevalence of diverging and chain motifs tends to increase correlation, while the converging motif makes no contribution to the average correlation. Moreover, we numerically show that variance of correlations across the network is largely determined by the frequency of the chain motif (see Figure 1) alone. Finally, we demonstrate potential effect of motif statistics on neural coding by showing how motif frequencies impact linear Fisher information. In particular, we find that the linear Fisher information is only affected by converging motif frequency (more information with given more converging motifs).

\section{Author details}

${ }^{1}$ Department of Applied Mathematics, University of Washington, Seattle, WA 98195, USA. ²Department of Mathematics, University of Houston, Houston, TX, 77204-5001, USA. ${ }^{3}$ Department of Biology and Biochemistry, University of Houston, Houston, TX, 77204-5001, USA. ${ }^{4}$ Program in Neurobiology and Behavior, University of Washington, Seattle, WA 98195, USA.

Published: 16 July 2012

\section{References}

1. Song S, Sjostrom PJ, Reigl M, Nelson S, Chklovskii DB: Highly nonrandom features of synaptic connectivity in local cortical circuits. PLOS Biol 2005, 3(3):e68.

2. Lindner B, Doiron B, Longtin A: Theory of oscillatory firing induced by spatially correlated noise and delayed inhibitory feedback. Phys Rev $E$ 2005, 72(6):061919.

3. Trousdale J, Hu Y, Shea-Brown E, Josić K: Impact of network structure and cellular response on spike time correlations. PLOS Biol, to appear.
4. Pernice V, Staude B, Cardanobile S, Rotter S: How structure determines correlations in neuronal networks. PLoS Comput Biol 2011, 7(5):e1002059.

5. Zhao L, Beverlin B, Neto T, Nykamp DQ: Synchronization from second order network connectivity statistics. Front Comput Neurosci 2011, 5:1-16.

6. Gerstner W, Kistler W: Spiking Neuron Models. Cambridge: Cambridge University Press; 2002.

\section{doi:10.1186/1471-2202-13-S1-P43}

Cite this article as: Hu et al:: Motif statistics and spike correlations in neuronal networks. BMC Neuroscience 2012 13(Suppl 1):P43.

\section{Submit your next manuscript to BioMed Central and take full advantage of:}

- Convenient online submission

- Thorough peer review

- No space constraints or color figure charges

- Immediate publication on acceptance

- Inclusion in PubMed, CAS, Scopus and Google Scholar

- Research which is freely available for redistribution 